\title{
Research on Artificial Spider Web Model for Farmland Wireless Sensor Network
}

\author{
Jun Wang, ${ }^{1}$ Song Gao $\mathbb{D}^{1},{ }^{1}$ Shimin Zhao, ${ }^{2}$ Guang Hu, ${ }^{1}$ Xiaoli Zhang, ${ }^{1}$ and Guowang Xie ${ }^{1}$ \\ ${ }^{1}$ College of Agriculture Equipment Engineering, Henan University of Science and Technology, Luoyang, Henan 471003, China \\ ${ }^{2}$ Production Technology Center, Luoyang Tobacco Company, Luoyang, Henan 471000, China
}

Correspondence should be addressed to Song Gao; gaosongsan@163.com

Received 13 March 2017; Revised 22 November 2017; Accepted 4 March 2018; Published 22 March 2018

Academic Editor: Ernestina Cianca

Copyright (C) 2018 Jun Wang et al. This is an open access article distributed under the Creative Commons Attribution License, which permits unrestricted use, distribution, and reproduction in any medium, provided the original work is properly cited.

\begin{abstract}
Through systematic analysis of the structural characteristics and invulnerability of spider web, this paper explores the possibility of combining the advantages of spider web such as network robustness and invulnerability with farmland wireless sensor network. A universally applicable definition and mathematical model of artificial spider web structure are established. The comparison between artificial spider web and traditional networks is discussed in detail. The simulation result shows that the networking structure of artificial spider web is better than that of traditional networks in terms of improving the overall reliability and invulnerability of communication system. A comprehensive study on the advantage characteristics of spider web has important theoretical and practical significance for promoting the invulnerability research of farmland wireless sensor network.
\end{abstract}

\section{Introduction}

Wireless sensor network can well support information perception in precision agriculture $[1,2]$. As an important part of IOT (Internet of Things), it can not only detect various environmental factors necessary for crop growth such as temperature, humidity, light intensity, soil moisture, soil nutrient, and carbon dioxide concentration through various sensors carried by the nodes deployed, but also provide advantages such as high precision, strong flexibility, and good reliability as compared to traditional wired means [3, 4]. Therefore, in the development of precision agriculture, it is of great significance to study wireless sensor network technology in depth. Agricultural monitoring is one of the WSN applications with important benefits to the farmers. Bogena et al. [5] used wireless sensor network for near-realtime monitoring of soil water content (SWC) variability in the field, which was not yet measured in an operational way at larger scales. Ruirui et al. [6] designed the node communication circuit based on atmegal28L MCU and CC1000 RF chip for acquisiting soil moisture/temperature/conductivity, air moisture/temperature, and illuminance. Díaz et al. [7] proposed a methodology consisting of a set of well-defined phases that can successfully guide the process of building monitoring applications for agricultural production based on WSN technology. The studies of wireless sensor network in farmland mainly focus on node development, energy saving strategy, networking protocol, and so on, but the invulnerability of the network is rarely analyzed and discussed. Nevertheless, the farmland wireless sensor network has the characteristics of super large scale, ultra-low cost, and complex topology. These characteristics impose more stringent requirements for network invulnerability. For example, in different growth periods, the dynamic change of planting height, density, and the density of branches and leaves has a significant impact on network transmission characteristics and link transmission quality, which is easy to cause link failures.

With complex farmland environment and numerous uncertain factors, the clustering method for traditional farmland wireless sensor network features poor dynamic performance and weak invulnerability [8]. In a clustering structure, all connections between cluster member nodes and the data center are single threaded [9]. Due to factors such as crop type conversion, change in growth period, difference in various components of precision operation, the environment 


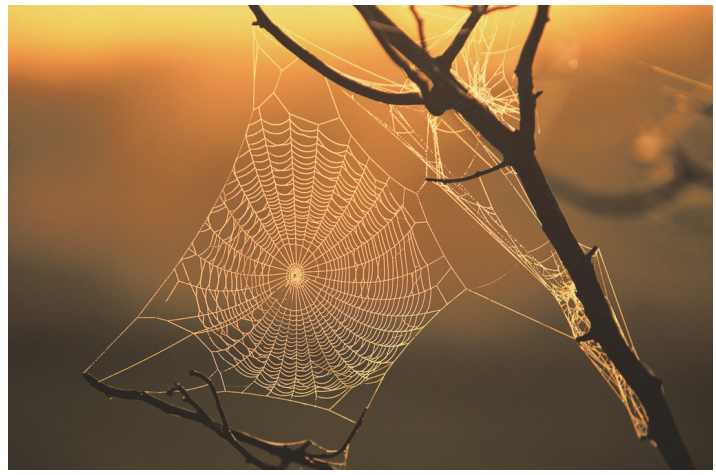

(a)

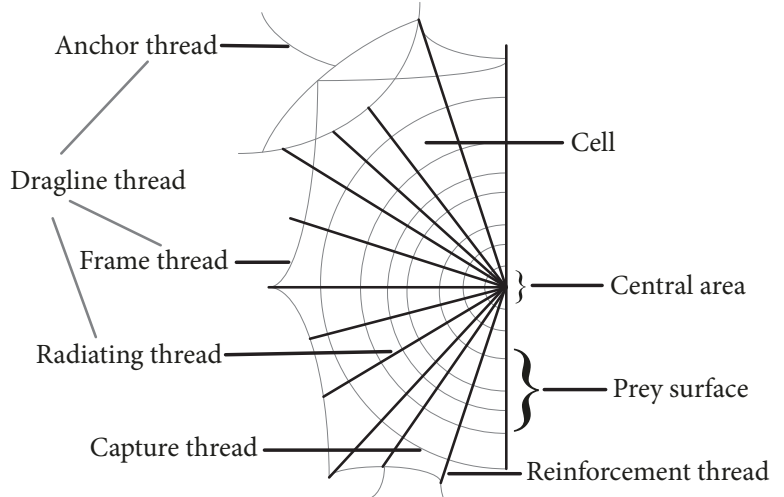

(b)

FIGURE 1: Orb spider web in the nature and its structural diagram.

and location of network nodes in the farmland often vary a lot and tend to cause communication link failure easily. In case of communication failure between the data center and some nodes, the entire network system will need to be reinitialized, or these nodes will lose communication all the time. Rerouting and invulnerability of a clustering structure are the key problems in restricting the application of farmland wireless sensor network.

With a super light structure, spider web in the nature can not only stick and catch a prey, but also communicate the information in the web effectively to the spider [10]. Its simple, elegant, and tough structure is a great enlightenment to farmland wireless sensor network. At present, many scholars at home and abroad have carried out helpful exploration in the construction principle, structure characteristic, and so forth of spider web [11]. The results show that a spider web can maintain strong and effective connectivity despite several damaged grids after impacted by a high load. This paper analyzes such problems as the structural characteristics of spider web, spider predation mechanism, and construction of artificial spider web communication topology to create the logical topology model of a bilayer hexagon artificial spider web. Xiaosheng et al. [12] use network structure and routing maintenance as the measures of communication reliability and employ factorization method in conjunction with the Markov probability model to carry out theoretical analysis on all terminal reliability of network communication, further proving the high communication reliability of the network structure of artificial spider web.

This paper uses orb web as the most representative type of spider web topology to study the structural characteristics and invulnerability of spider web, summarize and extract its characteristics of structural stability, and explore the possibility of combining the advantages of spider web such as network robustness and invulnerability with farmland wireless sensor network.

\section{Structure and Network Characteristics of Orb Spider Web}

After hundreds of millions of years of evolution, spider web has become a special structure integrating elegant, natural appearance and ultralight weight [13], as shown in Figure 1. Common orb spider web is oval with a certain degree of symmetry [14]. The general structure of a spider web is composed of dragline, capture, and reinforcement threads $[15,16]$. According to function, dragline thread can be further divided into three types: frame, anchor, and radiating threads. Frame thread is located in the periphery of a spider web, which composes the overall frame of the spider web, forms a variety of web surfaces, and determines the size and direction of the web body. Anchor thread serves as the web support that secures the entire spider web. Radiating thread, also known as radial thread, is radiating outward from the central area and connected with frame thread to maintain and support the stability of the entire spider web. Radiating thread has high ductility. Normally there are 16-46 radiating threads. This type of thread is able to communicate vibration information in the web for the spider to locate its prey. The central area is located in the center of an orb spider web's body, which is the core of the spider web.

With a spiral structure spinning outward from the central area, catch thread is the main spider silk for sticking and catching prey. Catch thread reflects the transmission of predation policy information. A cell is a small space enclosed by two adjacent radiating threads and two adjacent catch threads, and its size reflects the strength of the spider web; the smaller the cells are, the stronger the spider web generally is. Reinforcement thread is the silk a spider uses to secure the entire web according to the conditions of its actual position, which is random and adaptable to different environment.

Under the impact of various harsh natural and animal environments, spider web has evolved with characteristics that satisfy scientific laws. These characteristics are described as follows:

(1) The structure of spider web can be approximated as a special topological structure with center, which is composed of star topology and ring topology with elements crisscrossing each other in annular and radial forms; such kind of structure facilitates effective coverage of predation zone and collaborative monitoring of target prey; in addition, the density of cells determines the size of prey. 


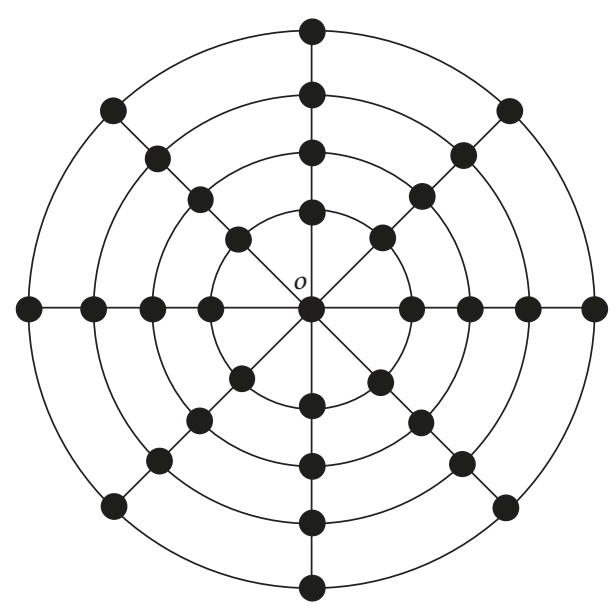

Figure 2: Artificial spider web model.

(2) The colliding or struggling signal of prey is transmitted to the central area through radiating and capture threads, and the spider senses the amplitude and frequency of the vibration signal through its legs, thus determining the exact location of the prey on the spider web.

(3) The structure of spider web features strong invulnerability; even if some cells are damaged, information can still be transmitted between the nodes of the spider web through other paths, which ensures effective connection; for the peripheral area of the spider web, the strength of the web body and the ability to communicate information are increased by weaving reinforcement threads.

(4) The strength and spacing of threads vary with their function, position, and importance.

The structure of an orb spider web is somewhat similar to the topological structure of a wireless sensor network. The central area of spider web is usually the collection point for various kinds of vibration information, which is similar to the sink node of a wireless sensor network that controls communication within the entire network. Since the vibration on spider web is mainly transmitted through radiating threads, these threads are considered as main routing path of the wireless sensor network, and since part of the vibration on the spider web can be transmitted through capture threads, these threads are considered as auxiliary path of the wireless sensor network, which is used when the main routing path fails or the traffic on it exceeds the threshold.

\section{Artificial Spider Web Model}

As inspired by the structural characteristics of orb spider web in the nature, a universally applicable definition of artificial spider web structure is given in this paper through observation, generalization, and summarization:

(1) An artificial spider web is composed of a star topology and several ring topologies. The subnodes of the ring topologies are also included in all the subnodes of the star topology.

(2) There must be a central area in the artificial spider web, which can be composed of one or more nodes and is able to establish a radial connection with all other subnodes.
(3) Inside the subnodes in the same layer of the artificial spider web, a tangential connection can be established between the subnodes, and each subnode can establish up to two direct tangential connection paths with other subnodes.

A typical artificial spider web is shown in Figure 2. Node $O$ is the central area of the entire spider web, which radiates outward in a star form and establishes direct or indirect radial connections with all other nodes. Each subnode is contained in the ring structure of the layer where it is located. This abstract topology is a good simulation of the structural characteristics of an orb spider web in the nature. Its core idea is to combine star topology with ring topology to form a mesh topology. The topological structure of spider web provides several backup links for communication in farmland wireless sensor network, thus greatly enhancing the reliability of communication.

To explain the structural characteristics of artificial spider web more clearly and to provide theoretical basis for further study of spider web, the main characteristic parameters of artificial spider web are defined as follows.

(1) $m$ is the number of spider web layers around Central Node $O$, that is, the number of concentric circle layers;

(2) $n$ is the number of spider web radial lines connected between Central Node $O$ and peripheral nodes, that is, the number of communication paths between Central Node $O$ and neighboring peripheral nodes;

(3) $p$ is the total number of nodes in the artificial spider web, and its relation with $m$ and $n$ is shown in the following equation:

$$
p=m \times n+1 ;
$$

(4) $R$ is the diameter of the spider web, which directly reflects the physical area covered by the spider web;

(5) $\theta_{n}$ is the sector angle of the spider web, that is, the included angle between two adjacent radial paths.

\section{Structural Model of Artificial Spider Web}

A sector cell in the spider web is selected for data analysis and the structure of this sector cell is determined. The first sector 
cell in the first layer of the spider web is selected for analysis, as shown in Figure 3 (Arc $A B$ is expressed in red).

Assume that the distance between Nodes $A, B$ and the spider web center is $R_{1}$; the included angle between
$A O$ and $B O$ is $\theta_{1}$; the included angle between $\operatorname{Arc} A B$ 's tangent on Point $A$ and $A O$ is $\varphi_{1}$; the radius of the corresponding circle of $\operatorname{Arc} A B$ is $r_{1}$, and the included angle is $\theta_{1}^{\prime}$.

$$
\begin{gathered}
\text { Then the area of Triangle } A B O \text { is. } S_{\triangle A B O}=\frac{1}{2} R_{1}{ }^{2} \sin \theta_{1} \\
\text { The area of Triangle } A B O^{\prime} \text { is. } \quad S_{\triangle A B O^{\prime}}=\frac{1}{2} r_{1}{ }^{2} \sin \theta_{1}^{\prime}
\end{gathered}
$$

The corresponding included angle of Arc $A B$ is. $\theta_{1}^{\prime}=2 \varphi_{1}+\theta_{1}-\pi$

$$
\text { The area of Sector } A B O^{\prime} \text { is. } S_{A B O^{\prime}}=\frac{\left(2 \varphi_{1}+\theta_{1}-\pi\right) \pi r_{1}^{2}}{2 \pi}
$$

The linear distance between Points $A$ and $B$ is. $l_{A B}=\sqrt{2 R_{1}{ }^{2}-2 R_{1}{ }^{2} \cos \theta_{1}}$

$$
l_{A B}=\sqrt{2 r_{1}^{2}-2 r_{1}^{2} \cos \left(2 \varphi_{1}+\theta_{1}-\pi\right)}
$$

The area of the selected sector cell is. $S_{A B O}=S_{\triangle A B O}+S_{A B O^{\prime}}-S_{\triangle A B O^{\prime}}$

(2):

The area of the selected sector cell can be obtained from

$$
\begin{aligned}
& S_{A B O}=\frac{1}{2} R_{1}^{2} \sin \theta_{1}+\frac{R_{1}^{2}-R_{1}^{2} \cos \theta_{1}}{2-2 \cos \left(2 \varphi_{1}+\theta_{1}-\pi\right)}\left[2 \varphi_{1}\right. \\
& \left.+\theta_{1}-\pi-\sin \left(2 \varphi_{1}+\theta_{1}-\pi\right)\right] .
\end{aligned}
$$

This is generalized to an artificial spider web model as shown in Figure 4, where tangential connection between subnodes in the same layer is not a standard circle, and the included angle between radial lines is not even. Assume that there are $m$ layers and $n$ tangential lines in the spider web structure; $\theta_{i}$ is the included angle between Radial Line $i$ and the previous radial line; $\varphi_{i j}$ is the included angle between the tangential line and radial line at a node on Radial Line $i$ in Layer $j ; R_{j}$ is the length of the corresponding radial line of Layer $j ; A_{i j}$ is the area of Cell $i$ in Layer $j$ of the spider web; and $S_{i j}$ is the area of sector cell. Thus it can be determined that the structural parameter of the entire artificial spider web can be expressed as $D=\left[i, j, \theta_{i}, \varphi_{i j}, R_{j}\right]$, where

$$
A_{i j}=\left\{\begin{array}{ll}
S_{i 1}, & j=1 \\
S_{i j}-S_{i(j-1)}, & 1<j \leq m
\end{array} \quad(1 \leq i \leq n) .\right.
$$

The optimal coverage problem of a typical artificial orb spider web is analyzed, in which the optimal coverage is the optimal coverage rate achieved with the minimum number of nodes at a given target monitored area. Assume that the maximum communication distance of the nodes is $L$, and the target monitored area is a $D \times D$ square, as shown in Figure 5 . It can be inferred that the structural model parameters of the artificial spider web with optimal network coverage should satisfy the following conditions:

$$
\begin{aligned}
\theta_{i}^{\prime} \times R_{m} & =L \\
n & =\operatorname{ceil}\left(\frac{2 \pi}{\theta_{1}^{\prime}}\right) \\
\theta_{1} & =\theta_{2}=\cdots=\theta_{n}=\frac{2 \pi}{n} \\
m & =\operatorname{ceil}\left(\frac{D}{2 L}\right),
\end{aligned}
$$

where ceil is a function that returns the smallest integer greater than or equal to the specified expression. Then the maximum coverage area of this typical artificial orb spider web is

$$
A_{1}=\pi R_{m}^{2}
$$

Now the coverage rate of the target monitored area is $P_{1}=$ $A_{1} / D^{2}$.

The coverage rate of this typical circular artificial spider web is compared with that of a polygon network as shown in Figure 6. When the polygon network reaches optimal coverage, its structural parameters should satisfy the following conditions:

$$
\begin{aligned}
& R_{m}=\sqrt{\left(\frac{D}{2}\right)^{2}+\left(\frac{L}{2}\right)^{2}} \\
& \theta_{i}^{\prime}=2 \arcsin \left(\frac{L}{R_{m}}\right)
\end{aligned}
$$




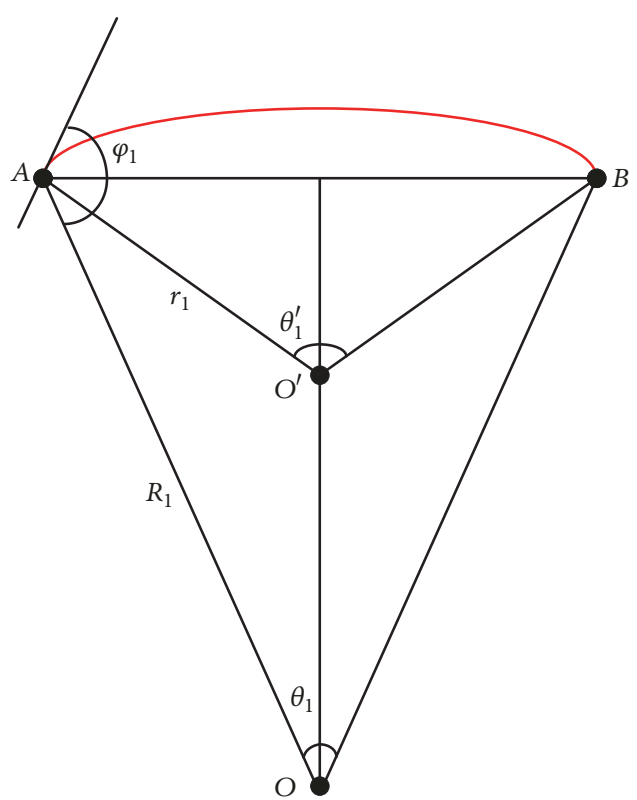

Figure 3: Sector cell.

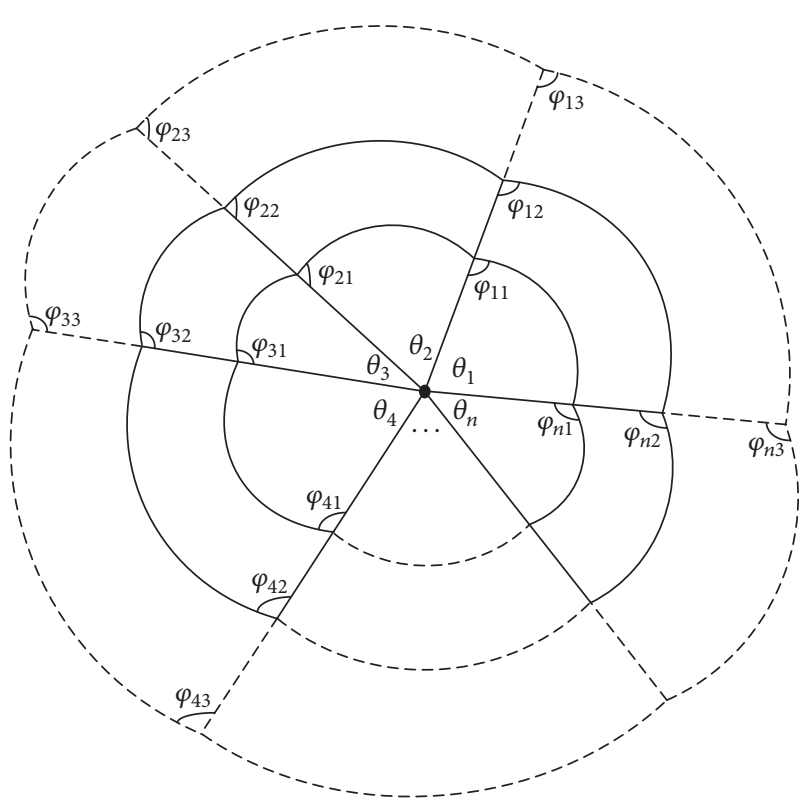

FIgURE 4: Structural model of artificial spider web.

$$
\begin{aligned}
& n=\operatorname{ceil}\left(\frac{2 \pi}{\theta_{1}^{\prime}}\right) \\
& \theta_{1}=\theta_{2}=\cdots=\theta_{n}=\frac{2 \pi}{n} \\
& m=\operatorname{ceil}\left(\frac{R_{m}}{L}\right) .
\end{aligned}
$$

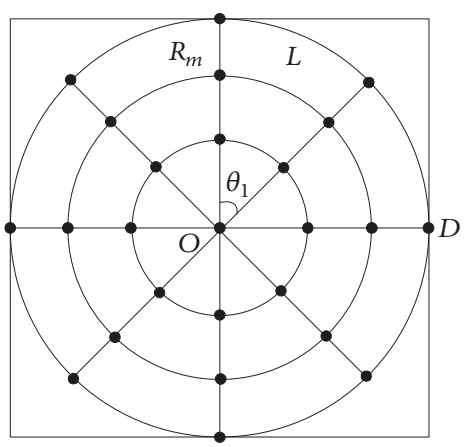

FIgURE 5: Area coverage structure of artificial spider web.

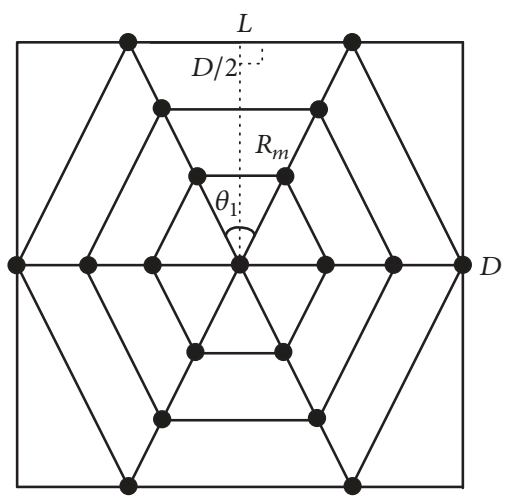

FIGURE 6: Area coverage structure of polygon network.

Then the maximum coverage area of this polygon network structure is

$$
A_{2}=\sum_{i=1}^{n} \frac{1}{2} R_{m}^{2} \sin \theta_{1}
$$

Now the coverage rate of the target monitored area is $P_{2}=$ $A_{2} / D^{2}$.

\section{Characteristic Parameters of Artificial Spider Web's Topological Structure}

The topological features of the artificial spider web conform to the characteristics of complex network, which make it possible to utilize and improve the related definitions in complex networks to describe the cobweb characteristics. Six characteristic parameters are defined to implement a statistical analysis of artificial spider web's topological structure.

5.1. Number of Multipaths. The direction of network path is defined as the transmission path along nodes in the same layer or from nodes in lower layer to upper layer, which must not be reversed. The total number of paths between Central Node $O$ and Arbitrary Node $a$ of an artificial spider web is expressed as

$$
L_{m}=(2 n-1)^{m},
$$




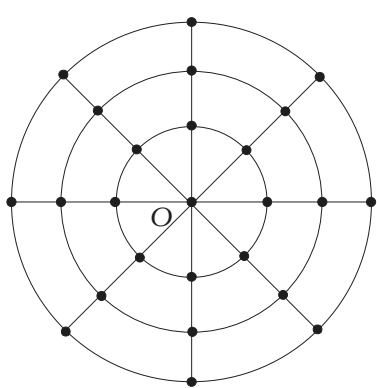

(a)

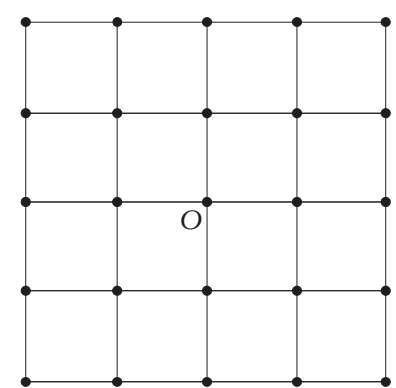

(b)

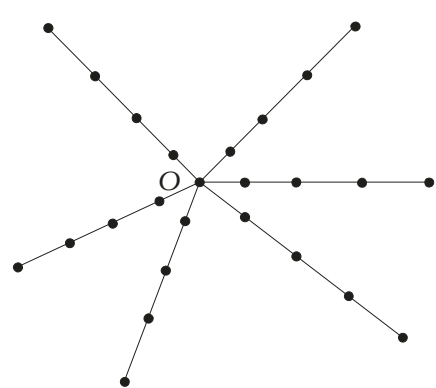

(c)

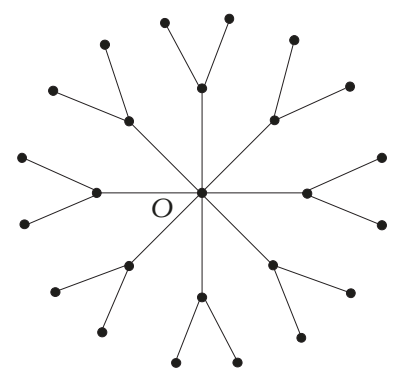

(d)

Figure 7: Network structure.

where $m$ is the layer where Node $a$ is located, $n$ is the number of radial lines, and $L_{m}$ is the total number of paths. The number of multipaths represents the redundancy of connections between nodes.

5.2. Hop. When any node reaches another node through a radial line or tangential line, it is called a hop. Assume that the number of hops between Central Node $O$ and Arbitrary Node $a$ of an artificial spider web is $h$; then

$$
\begin{aligned}
& h_{\text {min }}=m \\
& h_{\text {max }}=m \times q,
\end{aligned}
$$

where $m$ is the layer where Node $a$ is located, and $q$ is the number of tangential lines in the artificial spider web.

5.3. Node-Disjoint Paths. Except Central Node $O$ and the current node, there is no intersecting node or link on the path. The number of node-disjoint paths is used to indicate the robustness of the network. The more the number of node-disjoint paths, the stronger the network robustness. The number of node-disjoint paths is 3 .

5.4. Average Degree. Average number of communication lines connected by each node. The average degree expresses the overall importance of network nodes in a sense.

$$
k=\frac{2 M}{p},
$$

where $M$ is the number of edges and $p$ is the number of communication nodes.

5.5. Node Betweenness. The proportion of the number of shortest paths passing through Node $i$ in Network $G$ reflects the importance of Node $i$. The value of node betweenness depicts the amount of information that passes through the node during the transmission process.

$$
R_{1}(i)=\frac{\sum_{a \neq b \in G} N_{a, b}(i)}{\sum_{a \neq b \in G} N_{a, b}},
$$

where $\sum_{a \neq b \in G} N_{a, b}(i)$ is the number of shortest paths passing through Node $i \in G$ between Central Node $O$ and Arbitrary
Node $a$ in Network $G$ and $\sum_{a \neq b \in G} N_{a, b}$ is the total number of shortest paths between Central Node $O$ and Arbitrary Node $a$ in Network $G$. Spider web node betweenness is $R_{1}(i)=1$.

5.6. Node Cohesion. Connectivity of Local Network G composed of Node $i$ and other nodes directly connected with it.

$$
R\left(G_{i}\right)=\frac{k_{i}-1}{2 \sum_{a, b \in G} d_{a, b}(i)},
$$

where $k_{i}$ is the degree of Node $i$ and $d_{a, b}(i)$ is the minimum number of hops passing through Node $i$ between Arbitrary Node $a$ and Arbitrary Node $b$ in Network $G_{i}$. The node cohesion directly reflects the connectivity between the nodes in the network. The definition of node cohesion contains two parts of the numerator and the denominator. The molecular part is used to represent the ability of nodes to connect multiple communication lines, and the denominator part is applied to express the connectivity level between the node and the other nodes in the network.

The characteristic parameters of the topological structures of artificial spider web, grid network, star network, and tree network are compared, and the reliability, that is, invulnerability of the topological structures of these networks, is measured, as shown in Figure 7.

For different network structures, the same number of nodes ( 25 nodes in average) is selected, and the corresponding characteristic parameter values are calculated. The characteristic parameters of these four network structures were calculated by using graph theory toolbox (Matlab), namely, Matlab BGL V4.01. The characteristic parameter values of the topological structure of each network are shown in Table 1. It can be seen from the table that artificial spider web delivers significant advantages over other network structures in terms of number of multipaths, number of node-disjoint paths, average degree, node cohesion, and so forth. The advantage of multipaths of artificial spider web is greatest, which was up to 3375 .

In addition, the node cohesion of each layer of spider web is better than other network structures. However, the minimum number of hops is less than that of grid network and star network. The topological structure of spider web is so strong in connection stability that when the network is locally destroyed, it is more probable to maintain or restore 
TABLE 1: Characteristic parameter values of network topological structure.

\begin{tabular}{|c|c|c|c|c|}
\hline \multirow{2}{*}{ Characteristic parameter } & \multicolumn{4}{|c|}{ Network type } \\
\hline & Artificial spider web & Grid network & Star network & Tree network \\
\hline Number of multipaths & 3375 & 184 & 1 & 1 \\
\hline Minimum number of hops & 3 & 4 & 4 & 2 \\
\hline Number of node-disjoint paths & 3 & 2 & 1 & 1 \\
\hline Average degree & 3.84 & 3.2 & 1.92 & 1.92 \\
\hline Node betweenness & 1 & 0.67 & 0 or 1 & 0 or 1 \\
\hline \multirow{3}{*}{ Node cohesion } & Inner layer: 1.75 & Inner layer: 0.75 & Inner layer: 1.25 & Inner layer: 1.75 \\
\hline & Middle layer: 0.75 & Middle layer: 0.75 & Middle layer: 0.25 & Middle layer: 0.5 \\
\hline & Outer layer: 0.5 & Outer layer: 0.25 & Outer layer: 0 & Outer layer: 0 \\
\hline
\end{tabular}

Note. Inner layer is the 1st layer; middle layers include the 2nd layer to the second outermost layer; outer layer is the outermost layer.

its performance to an acceptable degree, thus offering greater invulnerability.

\section{Simulation Analysis}

To verify the invulnerability of artificial spider web model, MATLAB is used to conduct a corresponding simulation test. This paper adopts the network invulnerability evaluation method based on shortest message transmission time. Corresponding definitions are given below.

Definition 1. In an artificial spider network, $H_{\min }$ refers to the minimum number of hops between Node $i$ and Node $j$, then the shortest message transmission time between Node $i$ and Node $j$ is defined as

$$
T_{i j}=H_{\min } \times t,
$$

where $t$ is the duration of each hop.

Definition 2. For an artificial spider web model with total number of nodes being $p$, assuming that the central node disseminates an interest message to other nodes in the network through flooding routing, and ignoring the time required for outer layer nodes to transmit this message back to inner layer nodes, then the minimum average time for all the nodes in the target monitored area to receive this interest message is called the average shortest transmission time $T$; that is,

$$
T=\frac{\sum_{i=1}^{p} T_{i}}{p}
$$

where $T_{i}$ is the shortest time required for the interest message to be transmitted from the central node to Node $i$.

Definition 3. Invulnerability measurement indicator is

$$
\operatorname{Inv}=\left|\frac{T_{0}}{T}-1\right|,
$$

where $T_{0}$ is the average transmission time with a fully connected network and $T$ is the actual average transmission time. The Inv expressed in (16) is used to determine the invulnerability of network, and its value range is $[0,1]$. When

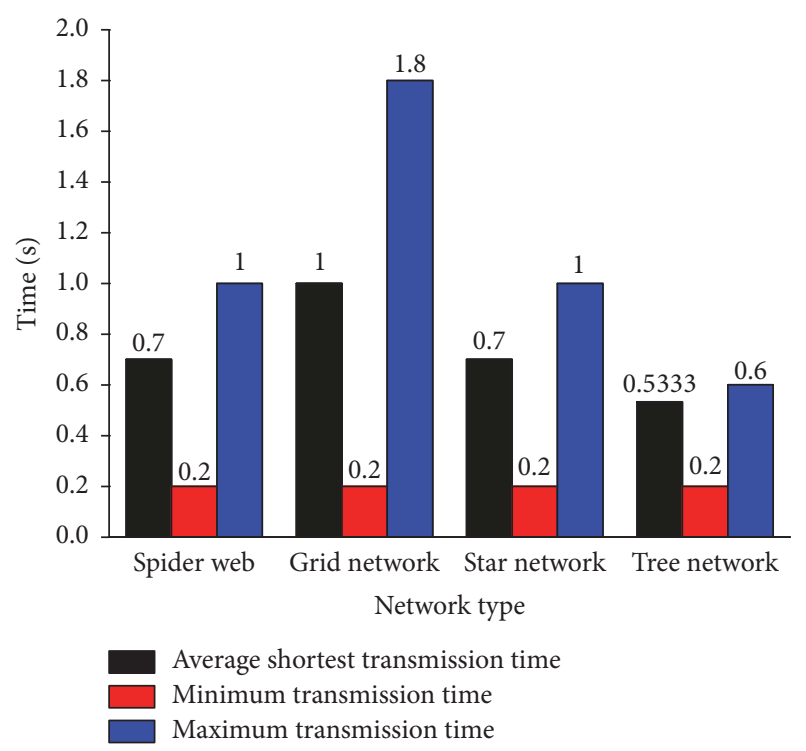

FIGURE 8: Transmission time of different network structures.

the network structure is complete, the values of $T_{0}$ and $T$ are exactly equal, then the value of invulnerability measurement indicator is 0 , which means the network is most stable. On the contrary, once the network structure is destroyed, the value of $T$ will inevitably increase. The better the connectivity of the network structure is, the smaller the actual average transmission time is, the lower the value of Inv is, and the stronger the invulnerability is after network destruction.

Four types of network structure in Figure 7 are analyzed using simulink software package to obtain the average shortest transmission time, minimum transmission time, and maximum transmission time, as shown in Figure 8. It is assumed that the network center spends $0.2 \mathrm{~s}$ to acquire the message for the network diffusion and the single hop time $t$ is $0.2 \mathrm{~s}$ in the whole simulation process of transmission time of different network structures. The measurements were done on an IBM ThinkPad T430 with a $2.5 \mathrm{GHz}$ CPU and 4 GB physical RAM. It can be seen from the figure that the three indicators of tree network are the shortest, those of star 
TABLE 2: Simulation results of artificial spider web.

\begin{tabular}{|c|c|c|c|}
\hline Number of layers & Number of remaining nodes & Average shortest transmission time (s) & Invulnerability indicator \\
\hline \multirow{5}{*}{1} & 5 & 0.7391 & 0.053 \\
\hline & 4 & 0.7818 & 0.105 \\
\hline & 3 & 0.8286 & 0.155 \\
\hline & 2 & 0.9400 & 0.255 \\
\hline & 1 & 1.0630 & 0.341 \\
\hline \multirow{5}{*}{2} & 5 & 0.7217 & 0.030 \\
\hline & 4 & 0.7455 & 0.061 \\
\hline & 3 & 0.7905 & 0.114 \\
\hline & 2 & 0.8400 & 0.167 \\
\hline & 1 & 0.9158 & 0.236 \\
\hline \multirow{5}{*}{3} & 5 & 0.7043 & 0.006 \\
\hline & 4 & 0.7091 & 0.013 \\
\hline & 3 & 0.7238 & 0.033 \\
\hline & 2 & 0.7400 & 0.054 \\
\hline & 1 & 0.7684 & 0.089 \\
\hline \multirow{5}{*}{4} & 5 & 0.6870 & 0.019 \\
\hline & 4 & 0.6727 & 0.041 \\
\hline & 3 & 0.6571 & 0.065 \\
\hline & 2 & 0.6400 & 0.094 \\
\hline & 1 & 0.6211 & 0.127 \\
\hline
\end{tabular}

network and spider web are the same in the middle level, and those of grid network are the longest.

When conducting invulnerability simulation for artificial spider web model, the parameters of artificial spider web model are set as follows: $m=4, n=6, p=25$, and single hop time $t=0.2 \mathrm{~s}$. Nodes are removed layer by layer from inside to outside, starting from the fewest number, and then the corresponding average shortest transmission time and invulnerability indicators after the network is damaged are counted. The simulation results are shown in Table 2. Meanwhile, the grid network, star network, and tree network in Figure 7 are also performed with the identical simulation analysis, which have the same total number of nodes as this spider web. The simulation results are depicted in Tables 3, 4 , and 5, respectively. By comparing the simulation results, the invulnerability performance of these networks can be obtained when these networks are damaged.

The data in Table 2 shows that the more the nodes in the same layer are removed, the greater the network invulnerability measurement indicator is, and the worse the network invulnerability is; between different layers, the smaller the number of layers where the node is located is, the greater the network invulnerability measurement indicator is after the node is removed, and the worse the network invulnerability is, indicating that the importance of inner layer nodes are higher than that of outer layer nodes. Through contrasting Table 2 with Tables 3, 4, and 5, it can be clearly seen that the overall invulnerability indicator of artificial spider web is significantly better than that of other network types in the node failure condition, which means that its topological structure has better reliability. Taking the outermost layer of network structure as an example, the maximum of invulnerability indicator of these network types is artificial spider web (0.127), grid network (1), star network (0.127), and tree network (0.263).

Due to the unpredictability of working environment, invulnerability is directly related to the stability, accuracy, and reliability in farmland wireless sensor network, and the realization of network function. It has become a key problem to enhance the invulnerability in the research of farmland wireless sensor network. The robustness and invulnerability of the network structure of artificial spider web can help improve the routing protocol of existing farmland wireless sensor network, optimize path selection result through fault tolerance for self-healing of network structure, balance the workload and data transmission on each path, reduce traffic congestion, prolong network lifetime, improve reliability, and avoid communication breakdown and network cavitation in farmland wireless sensor network.

This study uses spider web as the bionic research object and analyzes the structure characteristics and invulnerability performance of topological structure. Furthermore, the artificial spider web model is accurately built based on semantics and math formulas. It is found that there is a great similarity between the characteristics of the farmland wireless sensor network and spider web. The establishment, work, decisionmaking, and maintenance of wireless sensor network are similar to spider web, which provides the prerequisite for the application of the spider web to farmland wireless sensor network. Using the artificial spider web model described in this study, invulnerability mechanism of spider web can be computed and simulated. Such results can provide relevant information about the optimization of farmland wireless sensor network and can potentially be used as direct indicator 
TABLE 3: Simulation results of grid network.

\begin{tabular}{|c|c|c|c|}
\hline Number of layers & Number of remaining nodes & Average shortest transmission time (s) & Invulnerability indicator \\
\hline \multirow{7}{*}{1} & 7 & 0.9819 & 0.018 \\
\hline & 6 & 0.9709 & 0.030 \\
\hline & 5 & 0.9600 & 0.042 \\
\hline & 4 & 0.9589 & 0.043 \\
\hline & 3 & 0.9573 & 0.045 \\
\hline & 2 & 0.9552 & 0.047 \\
\hline & 1 & 0.9512 & 0.051 \\
\hline \multirow{15}{*}{2} & 15 & 0.9736 & 0.027 \\
\hline & 14 & 0.9667 & 0.034 \\
\hline & 13 & 0.9447 & 0.059 \\
\hline & 12 & 0.9223 & 0.084 \\
\hline & 11 & 0.9098 & 0.099 \\
\hline & 10 & 0.8976 & 0.114 \\
\hline & 9 & 0.8804 & 0.136 \\
\hline & 8 & 0.8720 & 0.147 \\
\hline & 7 & 0.8613 & 0.161 \\
\hline & 6 & 0.8548 & 0.170 \\
\hline & 5 & 0.8513 & 0.175 \\
\hline & 4 & 0.8400 & 0.191 \\
\hline & 3 & 0.8400 & 0.191 \\
\hline & 2 & 0.8400 & 0.191 \\
\hline & 1 & 0.2640 & 1.000 \\
\hline
\end{tabular}

TABLE 4: Simulation results of star network.

\begin{tabular}{|c|c|c|c|}
\hline Number of layers & Number of remaining nodes & Average shortest transmission time $(s)$ & Invulnerability indicator \\
\hline \multirow{5}{*}{1} & 5 & 0.9500 & 0.263 \\
\hline & 4 & 0.9500 & 0.263 \\
\hline & 3 & 0.9500 & 0.263 \\
\hline & 2 & 0.9500 & 0.263 \\
\hline & 1 & 0.9500 & 0.263 \\
\hline \multirow{5}{*}{2} & 5 & 0.6857 & 0.021 \\
\hline & 4 & 0.6667 & 0.050 \\
\hline & 3 & 0.6400 & 0.094 \\
\hline & 2 & 0.600 & 0.167 \\
\hline & 1 & 0.5333 & 0.313 \\
\hline \multirow{5}{*}{3} & 5 & 0.6818 & 0.027 \\
\hline & 4 & 0.6600 & 0.061 \\
\hline & 3 & 0.6333 & 0.105 \\
\hline & 2 & 0.6000 & 0.167 \\
\hline & 1 & 0.5571 & 0.256 \\
\hline \multirow{5}{*}{4} & 5 & 0.6870 & 0.019 \\
\hline & 4 & 0.6727 & 0.041 \\
\hline & 3 & 0.6571 & 0.065 \\
\hline & 2 & 0.6400 & 0.094 \\
\hline & 1 & 0.6211 & 0.127 \\
\hline
\end{tabular}

of the invulnerability of network topology and routing communication.

The next step of research should focus on addressing the application problem of the farmland wireless sensor network by the inheritance and innovation of invulnerability mechanism of spider web. A set of modeling and control algorithms will be proposed to improve the reliability of farmland wireless sensor network. Firstly, the topology management mechanism of static node deployment and dynamic cooperative scheduling should be established, which takes 
TABLE 5: Simulation results of tree network.

\begin{tabular}{|c|c|c|c|}
\hline Number of layers & Number of remaining nodes & Average shortest transmission time (s) & Invulnerability indicator \\
\hline \multirow{7}{*}{1} & 7 & 0.5333 & 0 \\
\hline & 6 & 0.5333 & 0 \\
\hline & 5 & 0.5333 & 0 \\
\hline & 4 & 0.5333 & 0 \\
\hline & 3 & 0.5333 & 0 \\
\hline & 2 & 0.5333 & 0 \\
\hline & 1 & 0.5333 & 0 \\
\hline \multirow{15}{*}{2} & 15 & 0.5304 & 0.005 \\
\hline & 14 & 0.5273 & 0.011 \\
\hline & 13 & 0.5238 & 0.018 \\
\hline & 12 & 0.5200 & 0.026 \\
\hline & 11 & 0.5158 & 0.034 \\
\hline & 10 & 0.5111 & 0.043 \\
\hline & 9 & 0.5059 & 0.054 \\
\hline & 8 & 0.5000 & 0.067 \\
\hline & 7 & 0.4933 & 0.081 \\
\hline & 6 & 0.4857 & 0.098 \\
\hline & 5 & 0.4769 & 0.118 \\
\hline & 4 & 0.4667 & 0.143 \\
\hline & 3 & 0.4545 & 0.173 \\
\hline & 2 & 0.4400 & 0.212 \\
\hline & 1 & 0.4222 & 0.263 \\
\hline
\end{tabular}

connectivity, cost, power consumption, and reliability as the constraint conditions. Secondly, the routing control strategy with multipath characteristics should be created for less hop number, less energy consumption, and better load balancing ability, under multiobjective optimization conditions. Therefore, in-depth study on the mechanism of combining the advantageous characteristics of spider web with farmland wireless sensor network has important theoretical and practical significance for promoting the research of self-healing routing and facilitating the development and application of farmland wireless sensor network.

\section{Conclusion}

This paper establishes a mathematical model of spider web, compares the characteristic parameters of spider web structure with other network structures, and conducts a simulation to verify the invulnerability of artificial spider web. As a result, the following conclusions are made:

(1) A universally applicable definition and mathematical model of artificial spider web structure are established to provide a theoretical basis for future research concerning spider web model.

(2) The characteristic parameters of artificial spider web structure are better than those of traditional grid, star, and tree networks; the networking structure of artificial spider web is better than that of traditional networks in terms of improving the overall reliability and invulnerability of communication system.

(3) Through systematic analysis of the structural characteristics and invulnerability of spider web, several research approaches to the survivable routing method that combines the advantageous characteristics of spider web with farmland wireless sensor network are offered.

\section{Conflicts of Interest}

The authors declare that they have no conflicts of interest.

\section{Acknowledgments}

This research activity described in this paper is jointly supported in part by National Natural Science Foundation of China (Grant no. 61771184), Key Research Project of Education Bureau of Henan Province, China (Grant no. 17A416002), and Key Scientific and Technological Project of Henan Province, China (Grant no. 172102210040).

\section{References}

[1] D. L. Ndzi, A. Harun, F. M. Ramli et al., "Wireless sensor network coverage measurement and planning in mixed crop farming," Computers and Electronics in Agriculture, vol. 105, pp. 83-94, 2014.

[2] X. Deqin, G. Zhichun, F. Jianzhao et al., "Design and experiment of wireless sensor networks for paddy field moisture monitoring," Transactions of the Chinese Society of Agricultural Engineering, vol. 27, no. 2, pp. 174-179, 2011.

[3] X. Kehui, X. Deqin, and L. Xiwen, "Smart water-saving irrigation system in precision agriculture based on wireless sensor network," Transactions of the Chinese Society of Agricultural Engineering, vol. 26, no. 11, pp. 170-175, 2010. 
[4] H. Yong, N. Pengcheng, and L. Fei, "Advancement and trend of internet of things in agriculture and sensing instrument," Transactions of the Chinese Society for Agricultural Machinery, vol. 44, no. 10, pp. 216-226, 2013.

[5] H. R. Bogena, M. Herbst, J. A. Huisman, U. Rosenbaum, A. Weuthen, and H. Vereecken, "Potential of wireless sensor networks for measuring soil water content variability," Vadose Zone Journal, vol. 9, no. 4, pp. 1002-1013, 2010.

[6] Z. Ruirui, Z. Chunjiang, C. Liping et al., "Design of wireless sensor network node for field information acquisition," Transactions of the Chinese Society of Agricultural Machinery, vol. 25, no. 11, pp. 213-218, 2009.

[7] S. E. Díaz, J. C. Pérez, A. C. Mateos, M.-C. Marinescu, and B. B. Guerra, "A novel methodology for the monitoring of the agricultural production process based on wireless sensor networks," Computers and Electronics in Agriculture, vol. 76, no. 2, pp. 252-265, 2011.

[8] S. Xiang, W. Baoguo, W. Huarui et al., "Topology Based Energy Efficient routing algorithm infarmland wireless sensor network," Transactions of the Chinese Society of Agricultural Machinery, vol. 46, no. 8, pp. 232-238, 2015.

[9] Y.-S. Miao, L. Yuan, H.-R. Wu, and Q.-X. Li, "Optimization of energy heterogeneous cluster-head selection in farmland WSN," Applied Mechanics and Materials, vol. 441, pp. 1010-1015, 2014.

[10] L. Zheng, M. Behrooz, and F. Gordaninejad, "A bioinspired adaptive spider web," Bioinspiration \& Biomimetics, vol. 12, no. 1, Article ID 016012, 2017.

[11] B. Mocanu, F. Pop, A. M. Mocanu, C. Dobre, and V. Cristea, "SPIDER: A Bio-inspired Structured Peer-to-Peer Overlay for Data Dissemination," in Proceedings of the 10th International Conference on P2P, Parallel, Grid, Cloud and Internet Computing, 3PGCIC 2015, pp. 291-295, Poland, November 2015.

[12] L. Xiaosheng, Z. Liang, Z. Yan et al., "Reliability analysis artificial cobweb structure for power-line communication of low-voltage distribution networks," Proceedings of the CSEE, vol. 32, no. 28, pp. 142-149, 2012.

[13] T. A. Blackledge, M. Kuntner, and I. Agnarsson, "The form and function of spider orb webs. Evolution from silk to ecosystems," Advances in Insect Physiology, vol. 41, pp. 175-262, 2011.

[14] S. P. Kelly, A. Sensenig, K. A. Lorentz, and T. A. Blackledge, "Damping capacity is evolutionarily conserved in the radial silk of orb-weaving spiders," Zoology, vol. 114, no. 4, pp. 233-238, 2011.

[15] A. M. T. Harmer, T. A. Blackledge, J. S. Madin, and M. E. Herberstein, "High-performance spider webs: Integrating biomechanics, ecology and behaviour," Journal of the Royal Society Interface, vol. 8, no. 57, pp. 457-471, 2011.

[16] Y. Hui, Y. Jialing, and L. Hua, "Simulation of prey stopping process by spider webs based on finite element method," Journal of Beijing University of Aeronautics and Astronautics, vol. 42, no. 2, pp. 280-286, 2016. 


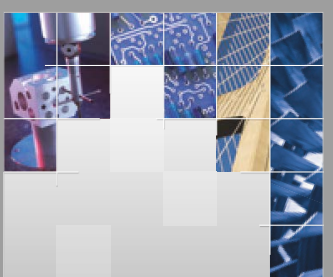

\section{Enfincering}
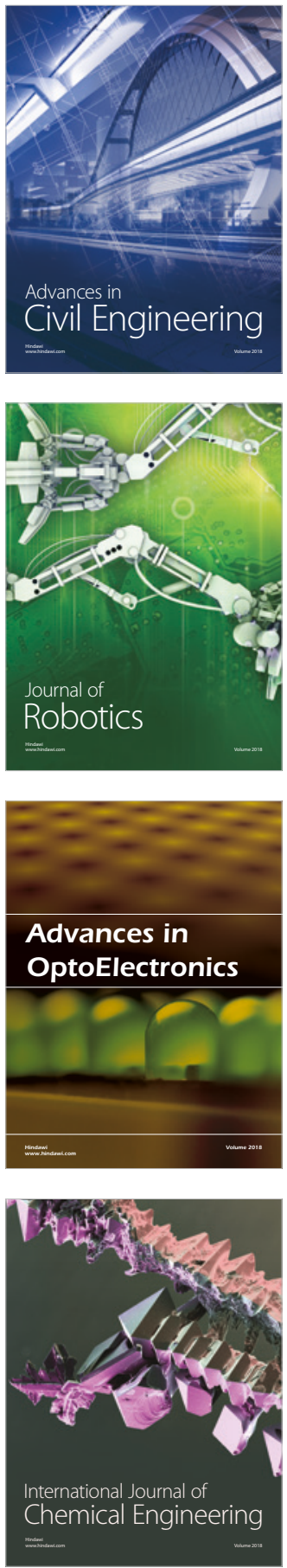

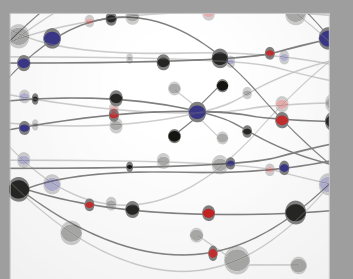

\section{Rotating \\ Machinery}

The Scientific World Journal

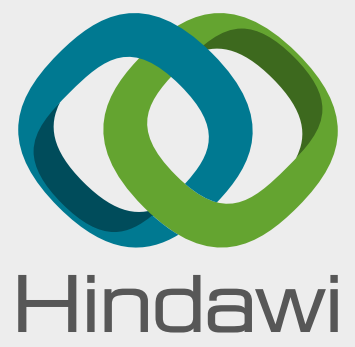

Submit your manuscripts at

www.hindawi.com
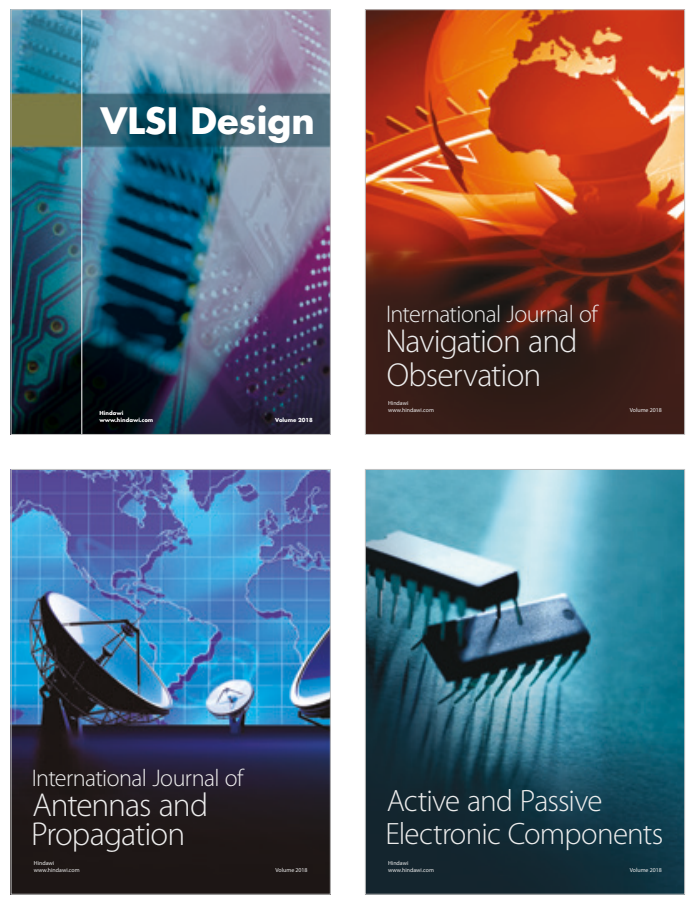
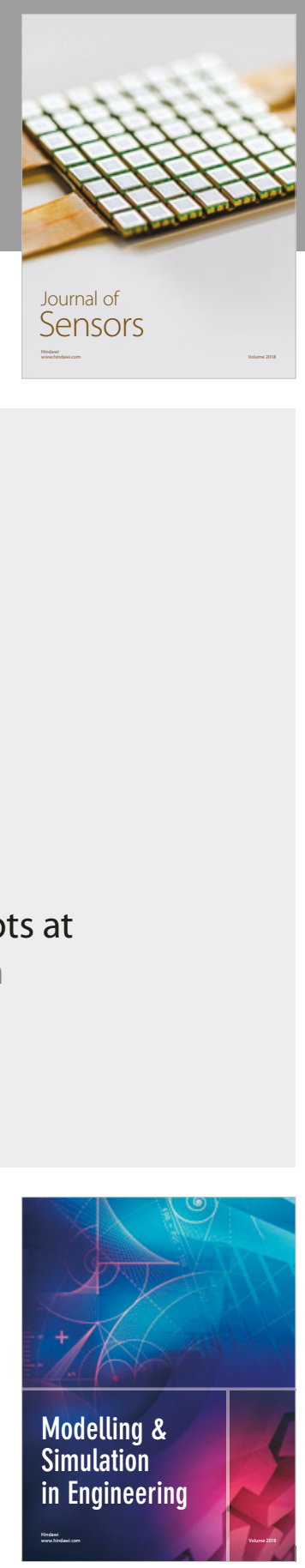

\section{Advances \\ Multimedia}
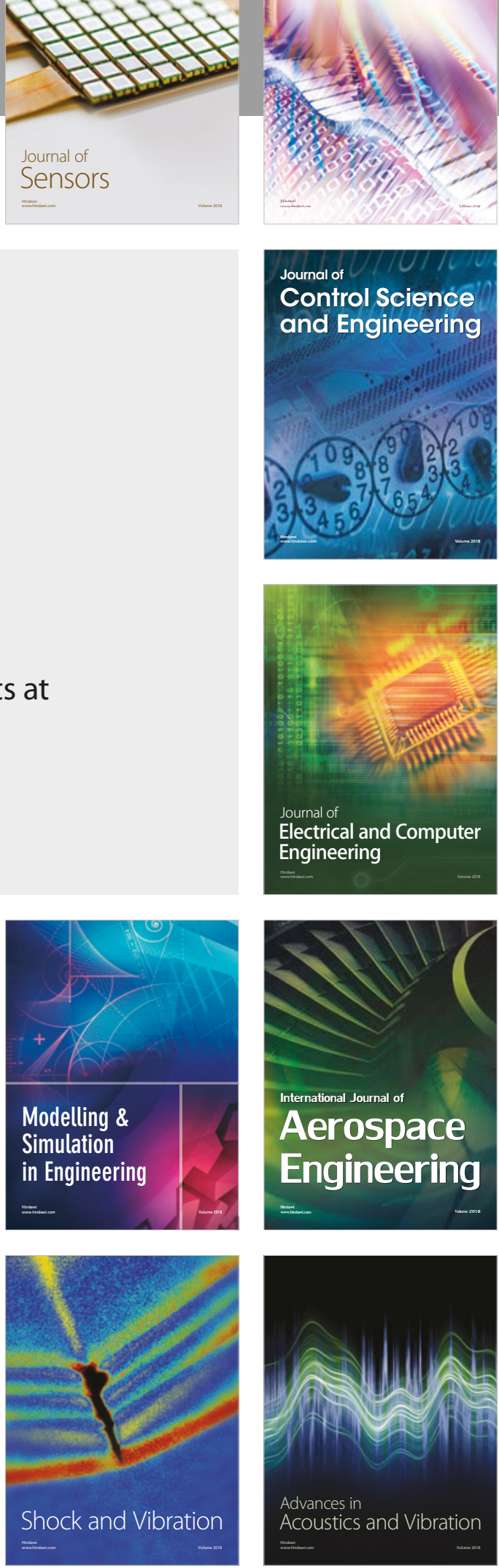